\title{
ONCTR: Optimum Number of Clusters and Transmission Range based Clustering in Wireless Sensor Networks
}

\author{
${ }^{1}$ Sumedha Sirsikar, ${ }^{2}$ Manoj Chandak \\ ${ }^{1}$ Scholar, Faculty of Engineering and Technology, Sant Gadge Baba Amaravati University, Maharashtra, India \\ ${ }^{2}$ Shri Ramdeobaba College of Engineering and Management, Nagpur, Maharashtra, India \\ Email: ${ }^{1}$ sirsikarsd@gmail.com, ${ }^{2}$ chandakmb@gmail.com
}

Received: $12^{\text {th }}$ Dec 2018, Accepted 17 ${ }^{\text {th }}$ March 18, Published: $30^{\text {th }}$ April 2018

\begin{abstract}
The field of Wireless Sensor Network (WSN) is striving for devising ways to minimize energy consumption. Clustering reduces energy consumption and increases scalability along with network lifetime. There is a need to identify appropriate number of clusters to balance traffic in network which is a challenging task for energy efficient WSN. Manually it is difficult to decide number of clusters. Finding optimum number of clusters to minimize energy consumption is the major issue in WSN. Existing algorithms find optimum number of clusters but not optimum transmission range. This paper contributes towards the aforesaid issue by proposing a novel method to find optimum number of clusters and a first attempt to find optimum transmission range. We report a new algorithm, where the number of clusters obtained from proposed method is compared with state-of-the-art methods. Extensive experiments are carried out and result comparison with state-of-the-art approaches demonstrate that our method shows significantly better performance. The analysis reveals that optimum number of clusters obtained by proposed method is less than state-of-the-art method. It is especially suitable for clustering in WSN.
\end{abstract}

Keywords: Optimal Clustering, Optimum Transmission Range, Self-organized Clustering

\section{Introduction}

In a sensor network, cost of communication between two nodes is much more than the cost of computation at each node where nodes are deployed randomly in a region. To provide a cost effective solution for minimizing communication overhead, nodes are grouped into various clusters. The challenges of energy efficient clustering are to find optimum number of nodes in a cluster, optimum number of clusters [1] and optimum position of $\mathrm{CH}$ [10][11]. In the design process of WSN, energy is the prime constraint. Optimum number of clusters is one of the ways to reduce energy consumption. However it has been observed that selecting random number of clusters gives non optimal results, increase in the number of clusters result into higher overall energy consumption and less number of clusters means more delay and more energy consumption. Hence there is need to address aforesaid issue. With this foothold,
We are proposing a new method called as Optimal Clustering (OC). Here Self-organized (SO) Clustering is used [17].

The main contributions of this paper are summarized as follows:

1. We presented a Square Root Methodology to find optimum number of clusters from number of deployed sensor nodes.

2. We proposed OC method to find optimum number of clusters and optimum transmission range.

3. We conducted extensive experiment on WSN to obtain optimum number of clusters to verify our proposal and also perform empirical comparison with state-of-the-art algorithms.

The rest of the paper is organized as follows: related work gives overview of various methods for optimal clustering and finding optimal number of clusters in WSN. The Square Root Methodology (SRM) for optimum number of clusters is explained. Then the proposed Optimal Clustering algorithm is described. Experimental results are summarized along with analytical findings and future work.

\section{Related Work}

During the design of WSN, a lot of research has focused on several clustering approaches with some constraint based on energy efficiency and lifetime of network [2]. The extensive work is carried out to select number of clusters in clustering techniques. This can be classified as connectivity-based, centroid-based and spectral graph [18]. The paper proposes connectivity-based optimal clustering algorithm i.e. clusters are formed within the transmission range of sensor nodes.

In the past two decades, several researchers attempted to find optimum number of clusters in WSN. In $\mathrm{LEACH}$, there are different numbers of clusters with different number of member nodes in various rounds. Hence energy consumption is also different in each round [3]. Hybrid Energy Efficient Distributed Clustering is based on iterative clustering process that uses the same approach to find optimum number of clusters as used in LEACH [4].

Gustafson-Kessel (GK) clustering algorithm forms clusters of equal area to achieve load balancing that uses fuzzy clustering to extend life of network [19]. Particle Swarm Optimization (PSO) algorithm for 
clustering gives unique optimal number of clusters for multi-cluster structure for WSN [7]. In Multiple Parameter based Clustering (MPC), optimum number of clusters is obtained analytically using cross layer approach [8] [16].

In K-means, number of clusters is to be given as input but that is not optimum [5]. Elbow method is used to calculate number of clusters. It uses percentage of variance that is function of cluster number. Elbow and K-means method (EBK) uses Elbow method to find number of clusters and cluster formation is as per K-means. Authors have not clearly mentioned how cost of optimal quality solution is calculated. EBK produces quality clusters and overall process improves energy consumption and network lifetime. It gives only optimum number of clusters [6] [14]. Hence there is a need to have such an algorithm that gives optimal clustering with optimum number of clusters as well as optimum transmission range. First principal component generated using Principal Component Analysis (PCA) is initialized as a cluster centroid in K-means. This reduces time taken for clustering and increases accuracy of the process [9]. L-method works on the principle of 'knee of a curve' that means point of maximum curvature where $\mathrm{x}$-axis represents number of clusters and $y$-axis represents evaluation metric. The evaluation metric is based on the clustering parameters such as distance, similarity, error and quality. Also L-method cannot work for one or two clusters [12]. Transmission range is one of the cluster quality parameter that is not evaluated against number of clusters. Fixed Optimal Clusters (FOC) algorithm analyzes an amount of optimal clusters based on the position of sink (far away or at the center of sensor field) and network model to extend lifetime of a network [13]. A fixed optimal cluster number is estimated along with $\mathrm{CHs}$ selection algorithm to utilize data correlation for aggregation [15]. Existing optimal clustering algorithm such as K-means, EBK and K-means PSO are compared as shown in the Table 1.

After comparison with algorithms mentioned in Table 1, we propose energy efficient Optimal Clustering which finds optimum number of clusters using OC method. Cluster Formation and $\mathrm{CH}$ Selection is dynamic and no need to recalculate position of $\mathrm{CH}$. However it has been evident from the Table 1 that the issue of finding optimum number of clusters needs to be emphasized. Hence we have been considering this as a major parameter in our methodology and algorithm explained in subsequent sections.

Table 1. Comparison of Existing Clustering Algorithms

\begin{tabular}{|l|l|l|l|l|l|}
\hline Algorithms & K-means & $\begin{array}{l}\text { Distributed } \\
\text { K-means }\end{array}$ & $\begin{array}{l}\text { Centralized } \\
\text { K-means }\end{array}$ & $\begin{array}{l}\text { EBK } \\
\text { Algorithm }\end{array}$ & $\begin{array}{l}\text { K-means } \\
\text { PSO }\end{array}$ \\
\hline $\begin{array}{l}\text { Optimum Number of } \\
\text { Cluster calculation }\end{array}$ & No & No & No & No \\
\hline $\begin{array}{l}\text { Optimum number of } \\
\text { cluster calculation } \\
\text { method }\end{array}$ & $\begin{array}{l}\text { Random } \\
\text { Selection of } \\
\text { Centroids }\end{array}$ & $\begin{array}{l}\text { Random } \\
\text { Selection of } \\
\text { Centroids }\end{array}$ & $\begin{array}{l}\text { Random } \\
\text { Selection of } \\
\text { Centroids }\end{array}$ & $\begin{array}{l}\text { Elbow } \\
\text { method }\end{array}$ & Static \\
\hline $\begin{array}{l}\text { Cluster Formation } \\
\text { and CH Selection }\end{array}$ & Random & $\begin{array}{l}\text { Distributed } \\
\text { (Participation } \\
\text { of each node } \\
\text { in the process) }\end{array}$ & $\begin{array}{l}\text { Controlled by } \\
\text { Central Node }\end{array}$ & Dynamic & Random \\
\hline $\begin{array}{l}\text { Recalculate position of } \\
\text { CH }\end{array}$ & $\begin{array}{l}\text { Yes } \\
\text { (Nearest }\end{array}$ & $\begin{array}{l}\text { Yes } \\
\text { (Nearest } \\
\text { Nodes to } \\
\text { Centroid) }\end{array}$ & $\begin{array}{l}\text { Yes } \\
\text { (Nearest } \\
\text { Nodes to } \\
\text { Centroid) }\end{array}$ & No & No \\
\hline Energy Efficient & Less & Medium & Medium & Medium & Less \\
\hline
\end{tabular}

\section{Square Root Methodology (SRM)}

There are several clustering algorithms that need initial value ' $C$ ' to choose number of clusters. To improve performance of WSN, value of ' $\mathrm{C}$ ' plays an important role. We have proposed the Square Root methodology to find optimum number of clusters which is described as follows:
Let $\left(\mathrm{X}_{1}, \mathrm{Y}_{1}\right)$ and $\left(\mathrm{X}_{2}, \mathrm{Y}_{2}\right)$ be the co-ordinates of two sensor nodes. Then the distance between two nodes is given by:

$$
\begin{aligned}
d & =\left[\left(X_{2}-X_{1}\right)^{2}+\left(Y_{2}-Y_{1}\right)^{2}\right]^{1 / 2} \\
\text { i.e., } & d^{2}=\left[\left(X_{2}-X_{1}\right)^{2}+\left(Y_{2}-Y_{1}\right)^{2}\right]
\end{aligned}
$$

Let, center of the sensor field is at origin of X-Y plane as shown in Fig. 1 (a). Hence, $\mathrm{d}^{2}=(\mathrm{X}-0)^{2}+(\mathrm{Y}-0)^{2}=\left(\mathrm{X}^{2}+\mathrm{Y}^{2}\right)$ 

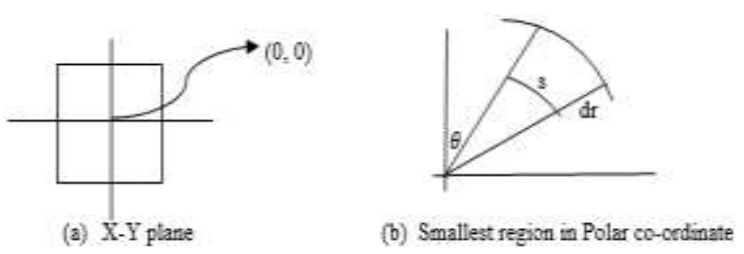

Figure 1. Calculation of area of sensor field

The distance between two sensor nodes i.e. distance from node to $\mathrm{CH}$ and $\mathrm{CH}$ to sink is denoted as $\mathrm{d}_{\mathrm{toCH}}$ and $\mathrm{d}_{\mathrm{toSink}}$.

From equation (1), $\mathrm{d}^{2}{ }_{\text {toCH }}$ or $\mathrm{d}_{\text {toSink }}^{2}=\left(\mathrm{X}^{2}+\mathrm{Y}^{2}\right)$ Average distance between all sensor nodes and cluster head over the region is calculated using double integrals as follows:

$\mathrm{d}_{\mathrm{toCH}}$ or $\mathrm{d}_{\mathrm{toSink}}=\int_{\mathrm{A}} \sqrt{\mathrm{X} * \mathrm{X}+\mathrm{Y} * \mathrm{Y}} \quad 1 / \mathrm{A} * \mathrm{dA}$--- (2)

Energy dissipated at the cluster head is given as follows:

$\mathrm{E}\left[\mathrm{d}^{2}{ }_{\mathrm{toCH}}\right]=\iint \mathrm{d}^{2} *$ density $* \mathrm{dx} \mathrm{dy}$

From equation (2) equation (3) is rewritten as follows:

$\mathrm{E}\left[\mathrm{d}^{2}{ }_{\mathrm{toCH}}\right]=\iint\left(\mathrm{X}^{2}+\mathrm{Y}^{2}\right) * \rho^{*}(\mathrm{X}, \mathrm{Y}) \mathrm{dx} \mathrm{dy}$

$\mathrm{E}\left[\mathrm{d}^{2}{ }_{\mathrm{toCH}}\right]=\iint\left(\mathrm{X}^{2}+\mathrm{Y}^{2}\right) * \rho *(\mathrm{X}, \mathrm{Y}) \mathrm{dA}$

where, area of region is $\mathrm{dA}=\mathrm{dx} * \mathrm{dy}$

Computing double integrals in rectangular coordinates is same as in polar co-ordinates.

Area of smallest rectangular region $\cong$ Area of smallest region on circle

Hence, $\mathrm{dA}=\mathrm{dx} d \mathrm{y}=\mathrm{r}^{*} \mathrm{dr} * \mathrm{~d} \theta$

Area of smallest region on circle $=a^{*} d r=r * d \theta * d r=$ $\mathrm{r} * \mathrm{dr} * \mathrm{~d} \theta$

i.e. as shown in Fig. $1(b), a=r * d \theta$

Equation of a region, $r^{2}=X^{2}+Y^{2}$

$\rho=$ sensor nodes density i.e. number of sensor nodes over the sensing area $=1 / \mathrm{A}$

The coverage area of a sensor node is considered to be a circle. Hence area of coverage $A=\pi r^{2}$ and therefore,

$r=\sqrt{A / \pi}$ where ' $r$ ' is the radius of coverage. Here, $\theta$ varies from ' 0 ' to ' $2 \pi$ ' and $r$ varies from ' 0 ' to ' $r$ ' that means $\sqrt{\mathrm{A} / \pi}$.

Hence, equation (4) becomes as follows:

$\mathrm{E}\left[\mathrm{d}^{2}{ }_{\text {toCH }}\right]=\int_{0}^{2 \pi} \int_{0}^{\sqrt{\mathrm{A} / \pi}} \mathrm{r}^{2}(1 / \mathrm{A}) \mathrm{rdr} \mathrm{d} \theta$

Assume, $\mathrm{M} \times \mathrm{N}$ is an area of a region in which ' $\mathrm{S}$ ' number of sensor nodes are deployed. Now equation

(5) becomes as follows:

$\mathrm{E}\left[\mathrm{d}^{2}{ }_{\mathrm{toCH}}\right]=\int_{0}^{2 \pi} \int_{0}^{\sqrt{\mathrm{M} * \mathrm{~N} / \pi}} 1 /(\mathrm{M} * \mathrm{~N}) \mathrm{r}^{3} \mathrm{dr} \mathrm{d} \theta$

Let $\mathrm{C}$ is the total number of clusters hence area of single cluster is $\mathrm{M}^{*} \mathrm{~N} / \mathrm{C}$, then equation (6) becomes:

$\mathrm{E}\left[\mathrm{d}^{2}{ }_{\mathrm{toCH}}\right]=\mathrm{C} /(\mathrm{M} * \mathrm{~N}) \int_{0}^{2 \pi} \int_{0}^{\sqrt{\mathrm{M} * \mathrm{~N} / \mathrm{C} * \pi}} \mathrm{r}^{3} \mathrm{dr} \mathrm{d} \theta$

$\mathrm{E}\left[\mathrm{d}^{2}{ }_{\mathrm{toCH}}\right]=\mathrm{C} /(\mathrm{M} * \mathrm{~N}) \int_{0}^{2 \pi}\left\{\left[\mathrm{r}^{4} / 4\right] \mid \sqrt{\mathrm{M} * \mathrm{~N} / \mathrm{C} * \pi}\right\} \mathrm{d} \theta$
$\mathrm{E}\left[\mathrm{d}^{2}{ }_{\mathrm{toCH}}\right]=\mathrm{C} /(\mathrm{M} * \mathrm{~N}) \int_{0}^{2 \pi}\left\{\left\{\left[\left(\mathrm{M}^{*} \mathrm{~N} / \mathrm{C}^{*} \pi\right)^{(1 / 2 * 4)}\right] / 4\right\}-0\right\} \mathrm{d} \theta$

$\mathrm{E}\left[\mathrm{d}^{2}{ }_{\mathrm{toCH}}\right]=\mathrm{C} /(\mathrm{M} * \mathrm{~N}) \int_{0}^{2 \pi}\left[\left(\mathrm{M}^{*} \mathrm{~N}\right)^{2} / 4 \mathrm{C}^{2 *} \pi^{*} \pi\right] \mathrm{d} \theta$

$\mathrm{E}\left[\mathrm{d}^{2}{ }_{\mathrm{toCH}}\right]=\mathrm{M} * \mathrm{~N} / 4 \mathrm{C}^{*} \pi^{*} \pi \int_{0}^{2 \pi} \mathrm{d} \theta$

$\mathrm{E}\left[\mathrm{d}^{2}{ }_{\mathrm{toCH}}\right]=\mathrm{M} * \mathrm{~N} / 4 \mathrm{C} * \pi * \pi[2 \pi-0]$

$\mathrm{E}\left[\mathrm{d}^{2}{ }_{\text {toCH }}\right]=\mathrm{M} * \mathrm{~N} / 2 \pi \mathrm{C}$

Sink is located at the center of $\mathrm{M} \times \mathrm{N}$ sensing area and the energy dissipated to the sink is as follows: $\mathrm{E}\left[\mathrm{d}^{2}{ }_{\text {toSink }}\right]=\int_{0}^{2 \pi} \int_{0}^{\sqrt{\mathrm{M} * \mathrm{~N} / \pi}}\left(\mathrm{X}^{2}+\mathrm{Y}^{2}\right)^{*} \rho^{*}(\mathrm{X}, \mathrm{Y}) \mathrm{dxdy}$

$\mathrm{E}\left[\mathrm{d}_{\text {toSink }}^{2}\right]=\int_{0}^{2 \pi} \int_{0}^{\sqrt{\mathrm{M} * \mathrm{~N} / \pi}} 1 /(\mathrm{M} * \mathrm{~N}) * \mathrm{r}^{3} * \mathrm{dr} \mathrm{d} \theta$

$E\left[d^{2}{ }_{\text {toSink }}\right]=2 \pi(1 / M * N) *\left\{r^{4} / 4 \mid \sqrt{M * N / \pi}\right\}$

$\mathrm{E}\left[\mathrm{d}^{2}{ }_{\text {toSink }}\right]=2 \pi(1 / \mathrm{M} * \mathrm{~N}) *\left[(\mathrm{M} * \mathrm{~N})^{2} / 4 \pi^{2}\right]$

$\mathrm{E}\left[\mathrm{d}^{2}{ }_{\text {toSink }}\right]=(\mathrm{M} * \mathrm{~N}) / 2 * \pi$

Initially, total number of hops is calculated to find total energy dissipated in the network.

The total number of hops for clustered network = number of hops (inter-cluster + intra-cluster)

$\mathrm{E}\{$ Hops $\}=\mathrm{E}_{\text {Total }}=\mathrm{E}_{\mathrm{CH}}+\mathrm{E}_{\text {non-CH }}$

$\mathrm{E}_{\text {Total }}$ is the total energy dissipated in the sensing area to transmit ' $b$ ' number of bits.

$\mathrm{E}_{\mathrm{CH}}=$ Energy required for $\{$ transmitter + data aggregation + receiver + amplifier $\}$

$\mathrm{E}_{\mathrm{CH}}=(\mathrm{S} / \mathrm{C}-1) * \mathrm{~b} *$Eelec $+\mathrm{S} / \mathrm{C} * \mathrm{~b} * \mathrm{E}_{\text {aggr }}+\mathrm{b} *$ Eelec $\quad+$ $\mathrm{b} * \mathcal{E}_{\mathrm{amp}} * \mathrm{~d}^{2}{ }_{\mathrm{toSink}}$

Where, ' $b$ ' is the number of bits transmitted, Eelec is radio dissipation energy constant for Transmitter and Receiver (per bit energy consumption),

$\varepsilon_{\text {amp }}$ is per bit amplified transmitting energy over the region, $E_{\text {aggr }}$ is per bit energy required for aggregation.

$\mathrm{E}_{\text {non-CH }}=$ Energy required for $\{$ receiver + amplifier $\}$

$\mathrm{E}_{\text {non- } \mathrm{CH}}=\mathrm{b}^{*} \mathrm{E}_{\text {elec }}+\mathrm{b} * \varepsilon_{\mathrm{amp}} * \mathrm{~d}_{\text {toCH }}^{2}$

$\mathrm{E}_{\text {cluster }}=\mathrm{E}_{\mathrm{CH}}+\{\mathrm{S} / \mathrm{C}-1\} \mathrm{E}_{\text {non-CH }}$

$\mathrm{E}_{\text {cluster }} \approx \mathrm{E}_{\mathrm{CH}}+\{\mathrm{S} / \mathrm{C}\} \mathrm{E}_{\mathrm{non}-\mathrm{CH}}$ 
Substitute the values from equation (10) and (11) into the equation (9).

$\mathrm{E}_{\text {Total }}=\mathrm{b}^{*}\left\{(\mathrm{~S} / \mathrm{C}-1)^{*} \mathrm{E}_{\text {elec }}+\mathrm{S} / \mathrm{C}^{*} \mathrm{E}_{\mathrm{aggr}}+\mathrm{E}_{\text {elec }}+\right.$ $\left.\varepsilon_{\text {amp }} * d_{\text {toSink }}^{2}\right\}+\mathrm{S} / \mathrm{C}\left\{\mathrm{E}_{\text {elec }}+\varepsilon_{\text {amp }} * \mathrm{~d}_{\text {toCH }}^{2}\right\} \quad \mathrm{E}_{\text {Total }}=\mathrm{b}$ $*\left\{2 \mathrm{~S} * \mathrm{E}_{\mathrm{elec}}+\mathrm{S} * \mathrm{E}_{\mathrm{aggr}}+\varepsilon_{\mathrm{amp}} *\left[\mathrm{C} * \mathrm{~d}_{\text {toSink }}^{2}+\mathrm{S} *\right.\right.$ $\left.\left.\mathrm{d}^{2}{ }_{\text {toCH }}\right]\right\}$

Number of optimum clusters $\mathrm{C}_{\text {opt }}$ can be obtained taking derivative of equation (12) w.r.t. total number of clusters $\mathrm{C}$ and equating it to zero.

$\delta \mathrm{E}_{\text {Total }} / \delta \mathrm{C}=0$

Substitute values for $\mathrm{d}^{2}{ }_{\text {toCH }}$ as well as $\mathrm{d}^{2}{ }_{\text {toSink }}$ in equation (12) from equation (7) and (8)

$$
\begin{aligned}
& \mathrm{d}^{2}{ }_{\text {toSink }}+\left.\frac{-\mathrm{S} * \mathrm{M} * \mathrm{~N}}{2 \pi * \mathrm{C} * \mathrm{C}}\right|_{\mathrm{C}=\mathrm{Copt}}=0 \\
& \mathrm{C}_{\mathrm{opt}}=\sqrt{\mathrm{S} * \mathrm{M} * \mathrm{~N} / 2 \pi} / \mathrm{d}_{\mathrm{toSink}} \\
& \mathrm{C}_{\mathrm{opt}}=\sqrt{S / 2 \pi} * \sqrt{\mathrm{M} * \mathrm{~N}} * \sqrt{2 \pi} / \sqrt{\mathrm{M} * \mathrm{~N}} \\
& \mathrm{C}_{\mathrm{opt}} \cong \sqrt{\mathrm{S}}
\end{aligned}
$$

Thus, the optimum number of clusters is based on the number of sensors in the entire network. Optimum Clustering

This section describes Optimal Clustering phases and OC model.

\section{Optimal Clustering Model}

Optimum Number of Clusters (ONC) model is described as follows:

$\mathrm{ONC}=\{\mathrm{SN}, \mathrm{NC}, \mathrm{TR}\}$

$\mathbf{S N}=$ Set of all nodes present in the network $=\{\mathrm{n} 1$, $\mathrm{n} 2, \mathrm{n} 3, \ldots$.

$\mathbf{N C}=$ Number of clusters $=\{\mathrm{nc} 1, \mathrm{nc} 2, \mathrm{nc} 3, \ldots\}$

TR =Transmission Range

Phases of Optimal Clustering (OC)

Optimal Clustering consists of five different phases which are described as follows:

Phase 1: Calculation of Optimum Number of Clusters

Sensor nodes are grouped in a non-overlapping clusters using SO clustering by varying transmission range. It gives number of clusters for a specific range. Graphs are plotted by taking transmission range at $\mathrm{x}$ axis and the number of clusters at y-axis. Two lines are drawn aligned to $\mathrm{x}$-axis and $\mathrm{y}$-axis which will cover maximum points on graph. First line is defined by two distinct points $(\mathrm{x} 1, \mathrm{y} 1)$ and $(\mathrm{x} 2, \mathrm{y} 2)$ and second line by $(x 3, y 3)$ and $(x 4, y 4)$. The intersection of two lines is $(\mathrm{x}, \mathrm{y})$ which satisfies equations of two lines and is given by equation (13) and (14):

$(\mathrm{x}-\mathrm{x} 1)(\mathrm{y} 2-\mathrm{y} 1)=(\mathrm{y}-\mathrm{y} 1)(\mathrm{x} 2-\mathrm{x} 1)$

$(\mathrm{x}-\mathrm{x} 3)(\mathrm{y} 4-\mathrm{y} 3)=(\mathrm{y}-\mathrm{y} 3)(\mathrm{x} 4-\mathrm{x} 3)$

The value of the $\mathrm{x}$ is the optimum transmission range for optimum number of clusters given by $y$.

\section{Phase 2: Cluster Head Selection}

Phase 1 gives optimum number of clusters and optimum transmission range. $\mathrm{CH}$ selection in $\mathrm{OC}$ is one on the basis of highest residual energy and nonzero number of neighbors otherwise node will be a cluster member.

Phase 3: Cluster Formation using optimum number of clusters and optimum range

The cluster formation is based on node distance. If node is in the optimum transmission range of a $\mathrm{CH}$ then it becomes member of a cluster.

\section{Phase 4: Data Transmission}

After formation of clusters, the member nodes sends data to their respective $\mathrm{CHs}$. Data aggregation is carried out at each $\mathrm{CH}$. Then all $\mathrm{CHs}$ send data to either $\mathrm{CH}$ or to Sink.

- Intra cluster

- Inter cluster (Only $\mathrm{CH}$ to $\mathrm{CH}$ )

- Finally to Sink

\section{Phase 5: Re-clustering}

After data transmission, the energy level of $\mathrm{CH}$ goes down as it consumes more energy and thus it cannot be continued as a cluster head. Hence, re-clustering takes place when residual energy of any $\mathrm{CH}$ goes below threshold value. Sensor node with highest residual energy is selected as $\mathrm{CH}$.

Experiments The sensor network is setup where all sensor nodes are stationary, homogeneous and can compute the approximate distance to other sensors. Sink is located at the center of sensor field and is static. NS-2 simulator is adopted to analyze optimal clustering algorithm. Table 2 shows the parameters used in setting up the wireless sensor network. The number of sensor nodes are considered as 50, 60, 70, $80,90,100,110$ and 120 . Transmission range is varied from $200 \mathrm{~m}$ to $500 \mathrm{~m}$ with the interval of $50 \mathrm{~m}$.

Analysis Optimal Clustering algorithm is run for various numbers of nodes and transmission ranges. Values for number of clusters are obtained using SO clustering and the graph is plotted for transmission ranges v/s number of clusters as shown in Figure 2. The knee point is determined and shown with a ring in Figures 2(a) to 2(h). For example, 50 sensor nodes are deployed and transmission range is varied. At each transmission range, number of clusters is obtained. Graph is plotted and lines are drawn such that it passes through maximum points on the graph. Point of intersection of these lines gives optimum number of clusters and optimum transmission range. For 50 nodes, the optimum transmission range obtained is $250 \mathrm{~m}$ and optimum number of clusters obtained is 7, as shown in Figure 2a. For 60 nodes, the optimum transmission range obtained is $200 \mathrm{~m}$ and optimum number of clusters obtained is 7 , as shown in Figure $2 b$. The same procedure is carried out for different number of nodes, and values are obtained. 
Table 2. Sensor Node Configuration Parameters

\begin{tabular}{|l|l|}
\hline Parameter & Value \\
\hline Network Area & $3400 \mathrm{~m} \times 1800 \mathrm{~m}$ \\
\hline Channel Type & Wireless \\
\hline Number of Nodes & $50,60, \ldots \ldots \ldots 120$ \\
\hline Initial Energy & $1000 \mathrm{Joules}$ \\
\hline Threshold Energy & $100 \mathrm{Joules}$ \\
\hline Transmission Range & $200 \mathrm{~m}, 250 \mathrm{~m}, \ldots \ldots . .500 \mathrm{~m}$ \\
\hline Transmitting and Receiving Power & 1 Watt \\
\hline Simulation Period & 100 Seconds \\
\hline
\end{tabular}
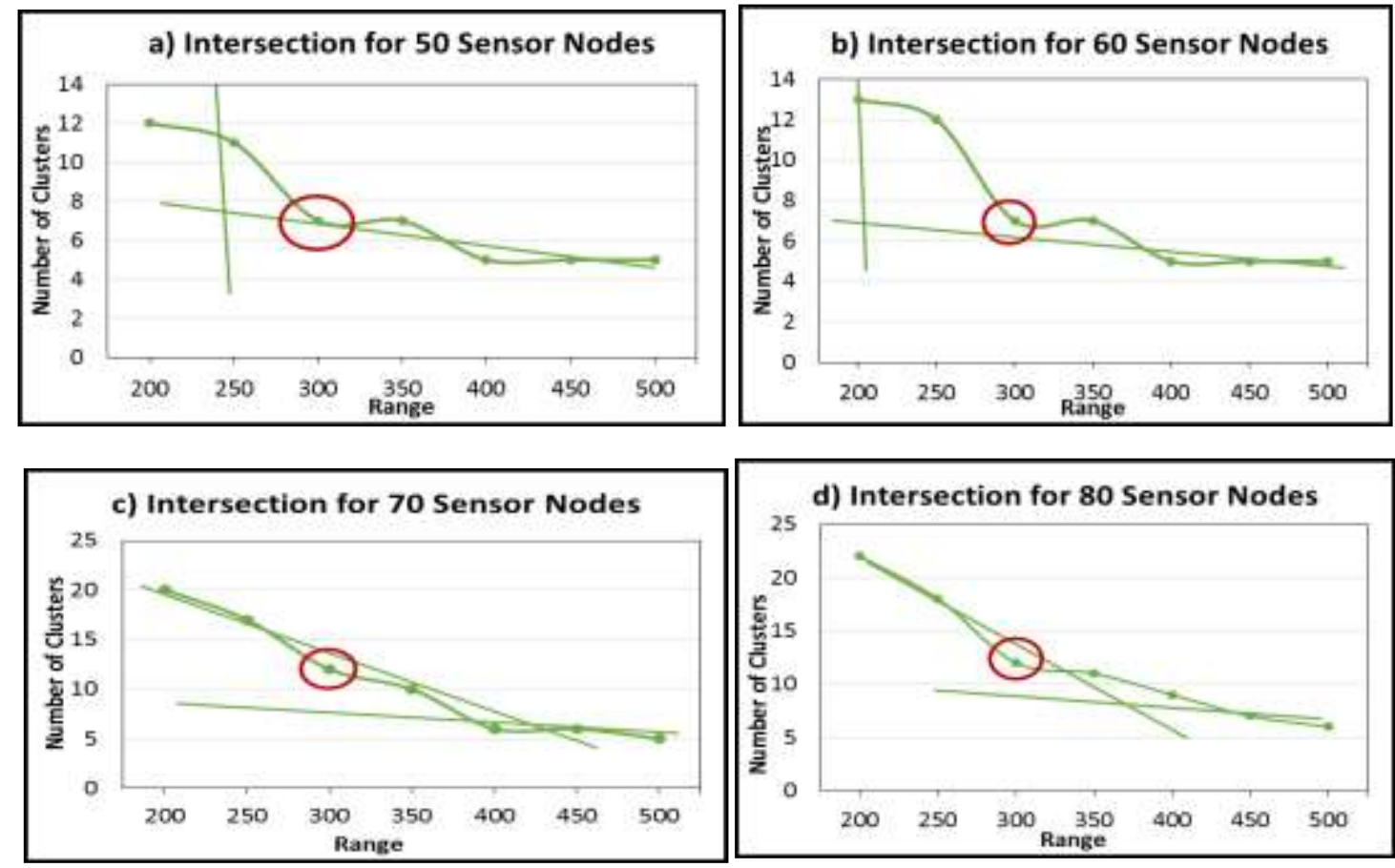

d) Intersection for $\mathbf{8 0}$ Sensor Nodes
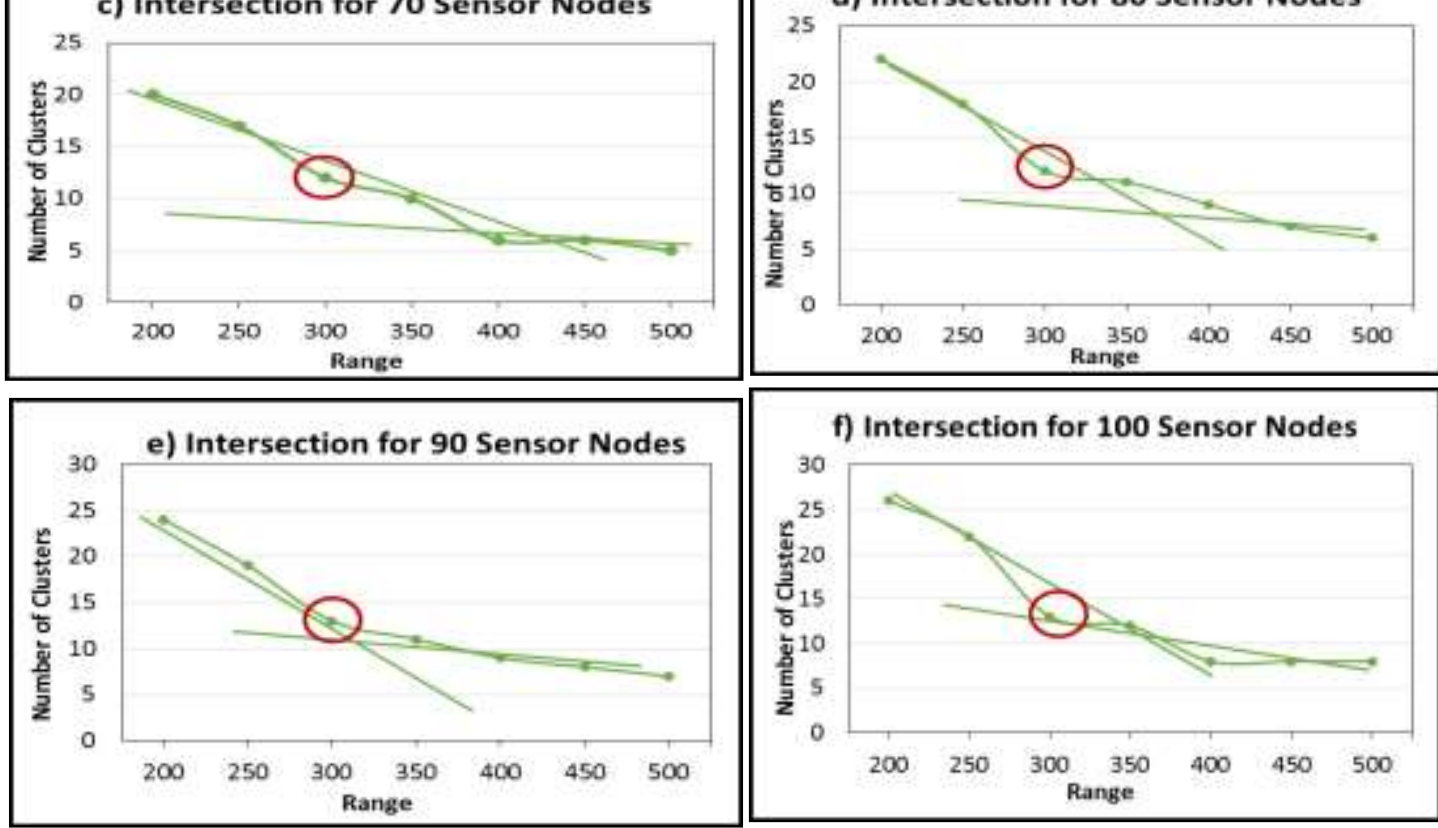

f) Intersection for $\mathbf{1 0 0}$ Sensor Nodes

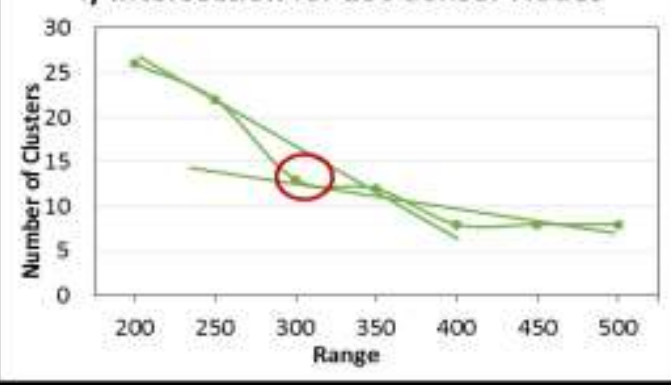

g) Intersection for $\mathbf{1 1 0}$ Sensor Nodes
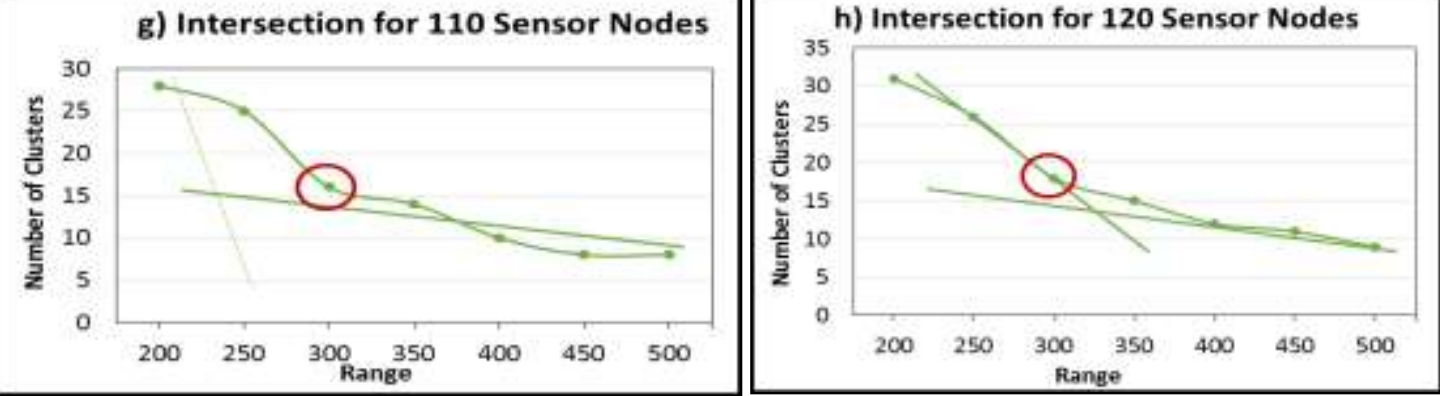

Figure 2. Calculation of ONC and Optimal Transmission range

Table 3. Number of Clusters Obtained by Self-organized Clustering 


\begin{tabular}{|l|c|c|c|c|c|c|c|c|}
\hline Y-axis & \multicolumn{9}{|c|}{ Number of Clusters } \\
\hline $\begin{array}{l}\text { Range } \\
\text { (X-axis) }\end{array}$ & $\begin{array}{c}\text { Number } \\
\text { of nodes } \\
\mathbf{5 0}\end{array}$ & $\begin{array}{c}\text { Number } \\
\text { of nodes } \\
\mathbf{6 0}\end{array}$ & $\begin{array}{c}\text { Number } \\
\text { of nodes } \\
\mathbf{7 0}\end{array}$ & $\begin{array}{c}\text { Number } \\
\text { of nodes } \\
\mathbf{8 0}\end{array}$ & $\begin{array}{c}\text { Number } \\
\text { of nodes } \\
\mathbf{9 0}\end{array}$ & $\begin{array}{c}\text { Number } \\
\text { of nodes } \\
\mathbf{1 0 0}\end{array}$ & $\begin{array}{c}\text { Number } \\
\text { of nodes } \\
\mathbf{1 1 0}\end{array}$ & $\begin{array}{c}\text { Number } \\
\text { of nodes } \\
\mathbf{1 2 0}\end{array}$ \\
\hline 200 & 12 & 13 & 20 & 22 & 24 & 26 & 28 & 31 \\
\hline 250 & 11 & 12 & 17 & 18 & 19 & 22 & 25 & 26 \\
\hline 300 & 7 & 7 & 12 & 12 & 12 & 13 & 16 & 18 \\
\hline 350 & 7 & 7 & 10 & 11 & 11 & 12 & 14 & 15 \\
\hline 400 & 5 & 5 & 6 & 9 & 9 & 8 & 10 & 12 \\
\hline 450 & 5 & 5 & 6 & 7 & 8 & 8 & 8 & 11 \\
\hline 500 & 5 & 5 & 5 & 6 & 7 & 8 & 8 & 9 \\
\hline
\end{tabular}

Table 3, gives number of clusters obtained by Selforganized Clustering. The lines are drawn on the curve aligned to $\mathrm{x}$-axis and $\mathrm{y}$-axis passing through maximum points on the curve. The $\mathrm{x}$ and $\mathrm{y}$ coordinate of the intersection of this two lines is the optimum range and optimum number of clusters obtained for OC method respectively as shown in Figure 2(a) to 2 (h). Table 4 gives Comparison of Number of Clusters and Range for k-means and OC Method. Table 5 gives Comparison of Number of Clusters and Range for Elbow and OC Method.

Table 4. Number of Clusters and Range for k-means and OC Method

\begin{tabular}{|c|c|c|c|c|}
\hline \multirow{2}{*}{$\begin{array}{c}\text { Number of } \\
\text { Sensor Nodes }\end{array}$} & \multicolumn{2}{|c|}{ k-means } & \multicolumn{2}{|c|}{ OC Method } \\
\hline & $\begin{array}{c}\text { Number of } \\
\text { clusters }\end{array}$ & Transmission Range & $\begin{array}{l}\text { Optimum } \\
\text { number of } \\
\text { clusters }\end{array}$ & Optimum Range \\
\hline 50 & 11 & 250 & 7 & 250 \\
\hline 60 & 13 & 200 & 7 & 200 \\
\hline 70 & 6 & 450 & 8 & 425 \\
\hline 80 & 10 & 350 & 7 & 366 \\
\hline 90 & 12 & 350 & 10 & 337 \\
\hline 100 & 9 & 400 & 13 & 383 \\
\hline 110 & 25 & 250 & 14 & 250 \\
\hline 120 & 15 & 350 & 14 & 337 \\
\hline
\end{tabular}

Table 5. Number of Clusters and Range for Elbow and OC Method

\begin{tabular}{|c|c|c|c|c|}
\hline \multirow{2}{*}{$\begin{array}{c}\text { Number of } \\
\text { Sensor Nodes }\end{array}$} & \multicolumn{2}{|c|}{ Elbow Method } & \multicolumn{2}{c|}{ OC Method } \\
\cline { 2 - 5 } & $\begin{array}{c}\text { Number of } \\
\text { clusters }\end{array}$ & Transmission Range & $\begin{array}{c}\text { Optimum } \\
\text { number of } \\
\text { clusters }\end{array}$ & Optimum Range \\
\hline 50 & 11 & 250 & 7 & 250 \\
\hline 60 & 13 & 200 & 7 & 200 \\
\hline 70 & 6 & 450 & 8 & 365 \\
\hline 80 & 9 & 350 & 10 & 337 \\
\hline 90 & 11 & 350 & 13 & 383 \\
\hline 100 & 8 & 400 & 14 & 250 \\
\hline 110 & 25 & 250 & 14 & 337 \\
\hline 120 & 15 & 350 & 7256 \\
\hline
\end{tabular}




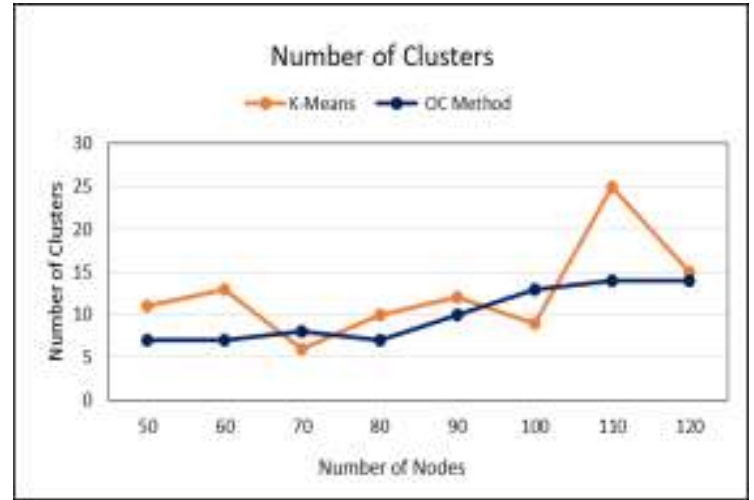

Figure 3. Comparison of Number of Clusters for k-means and OC Method

Graph of optimum number of clusters obtained from $\mathrm{k}$-means and OC method is plotted as shown in Figure 3. Graph of optimum number of clusters obtained from Elbow method and OC method is plotted as shown in Figure 4. X-axis represents number of nodes and $\mathrm{Y}$-axis represents number of clusters. It shows that the OC method gives optimum number of clusters as compared to k-means and Elbow method. Square Root Method gives only Optimum number of clusters (which is approximately equal to square root of total number of sensor nodes) and not Optimum transmission range. For example, for 50 nodes, 7 clusters are obtained so on and for 120 , we get 11 clusters.

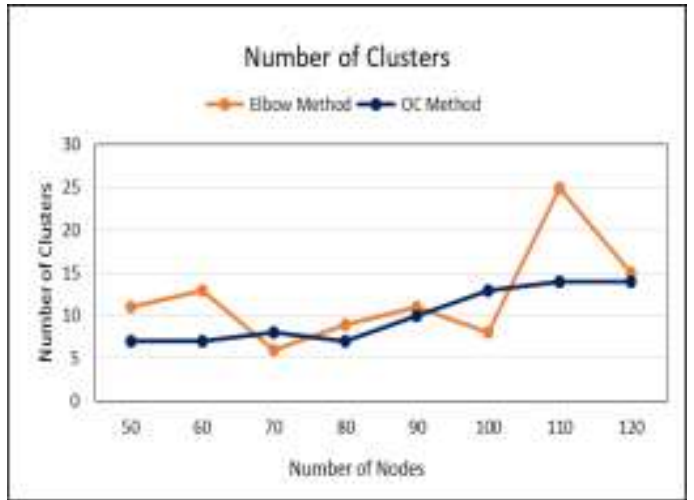

Figure 4. Comparison of Number of Clusters for Elbow and OC Method

Results for Average Energy Consumption and Network Lifetime

K-means and Elbow method are implemented as in [5] [6]. Table 5 gives values of Average Energy Consumption for K-Means, Elbow and OC Method. Fig. 5 and Fig. 7 show comparison of Average Energy Consumption and Network Lifetime for K-Means and OC Method respectively. Table 6 gives values of Network Lifetime for K-Means, Elbow and OC Method respectively.

Fig. 6 and Fig. 8 show comparison of Average Energy Consumption and Network Lifetime for Elbow and OC Method respectively. OC Method gives optimum number of clusters and hence results for energy and network lifetime are better as compared to K-Means and Elbow method.

Table 6. Average Energy Consumption and Network Lifetime

\begin{tabular}{|c|c|c|c|c|c|c|}
\hline & \multicolumn{2}{|c|}{ Output of OC } & \multicolumn{2}{c|}{ Output of k-means } & \multicolumn{2}{c|}{ Output of Elbow } \\
\hline $\begin{array}{c}\text { Numb } \\
\text { er of } \\
\text { Nodes }\end{array}$ & $\begin{array}{c}\text { Average Energy } \\
\text { Consumption } \\
\text { (Joule) }\end{array}$ & $\begin{array}{c}\text { Network } \\
\text { Lifetime } \\
\text { (second) }\end{array}$ & $\begin{array}{c}\text { Average } \\
\text { Energy } \\
\text { Consumption } \\
\text { (Joule) }\end{array}$ & $\begin{array}{c}\text { Network } \\
\text { Lifetime } \\
\text { (second) }\end{array}$ & $\begin{array}{c}\text { Average Energy } \\
\text { Consumption } \\
\text { (Joule) }\end{array}$ & $\begin{array}{c}\text { Network } \\
\text { Lifetime } \\
\text { (second) }\end{array}$ \\
\hline 120 & 17.99 & 3612.83 & 26.07 & 3004.58 & 18.38 & 3534.80 \\
\hline 110 & 11.21 & 6331.21 & 23.55 & 5381.53 & 11.21 & 6331.21 \\
\hline 100 & 21.02 & 2806.80 & 26.98 & 2343.30 & 22.85 & 2756.83 \\
\hline 90 & 15.87 & 3464.56 & 24.87 & 2811.90 & 17.23 & 3308.12 \\
\hline 80 & 19.39 & 3144.46 & 27.24 & 2920.98 & 22.50 & 3436.44 \\
\hline 70 & 23.02 & 2735.87 & 27.83 & 2217.99 & 24.90 & 2609.41 \\
\hline 60 & 5.97 & 6526.90 & 17.85 & 5547.86 & 5.975 & 6526.90 \\
\hline 50 & 8.85 & 4405.63 & 20.49 & 3744.79 & 8.85 & 4405.64 \\
\hline
\end{tabular}




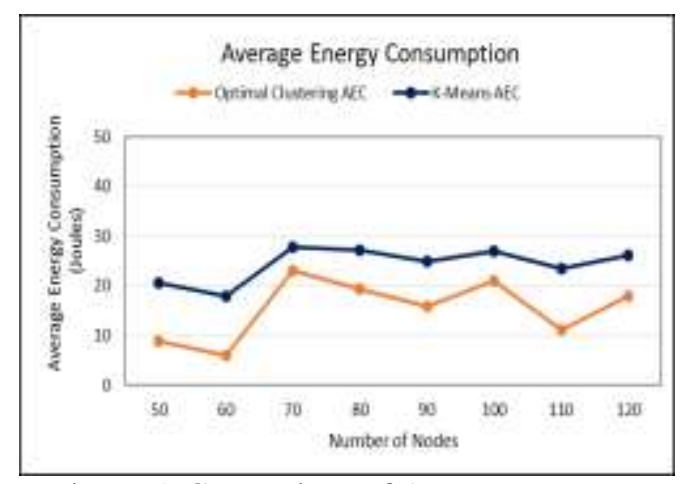

Figure 5. Comparison of Average Energy Consumption for k-means and OC Method

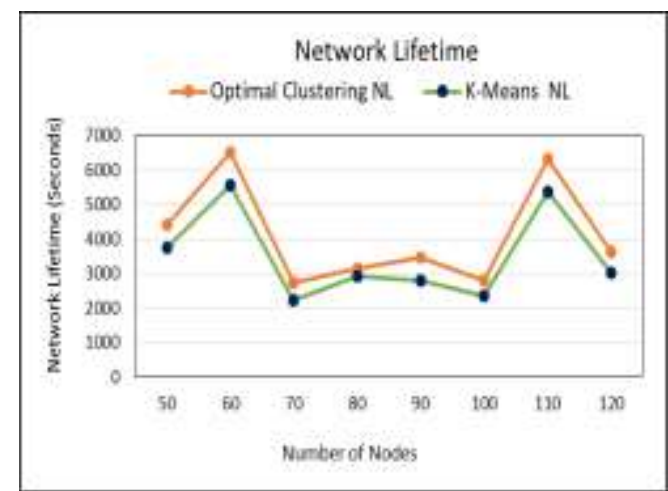

Figure 7. Comparison of Network Lifetime for k-means and OC Method

\section{Conclusion and Future Work}

An Optimal Clustering algorithm uses SelfOrganized Clustering based on the attributes like node degree, distance and residual energy. Number of clusters for k-means and Elbow method are obtained and compared with OC method. OC method gives Optimum number of clusters and Optimum transmission ranges. Optimum number of clusters obtained from Square Root Method is approximately equal to square root of total number of sensor nodes. $\mathrm{k}$-means gives random number of clusters which is not optimum and there is need to recalculate position of $\mathrm{CH}$ which is not required in OC method. OC performs better in terms of energy and network lifetime. Average Energy Consumption in OC is reduced by $1.23 \%$ as compared to $\mathrm{k}$-means and $0.31 \%$ as compared to Elbow Method. Similarly, Network lifetime is increased by $18.93 \%$ as compared to k-means and $4.62 \%$ as compared to Elbow Method.

In future, mobile nodes and sink can be introduced in the sensor network to have more energy efficient WSN.

\section{References}

1. Rahmani Naeim, HeliaKousha, Ladan Darougaran and Farhad Nematy, CAT: The new clustering algorithm based on two-tier network topology for energy balancing in wireless sensor networks, International Conference on

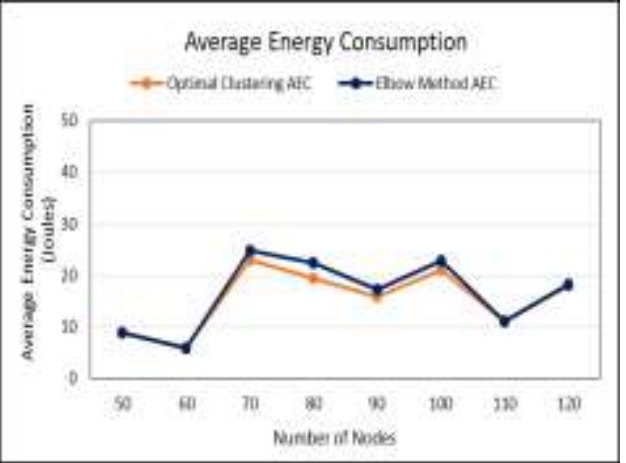

Figure 6. Comparison of Average Energy

Consumption for Elbow and OC Method

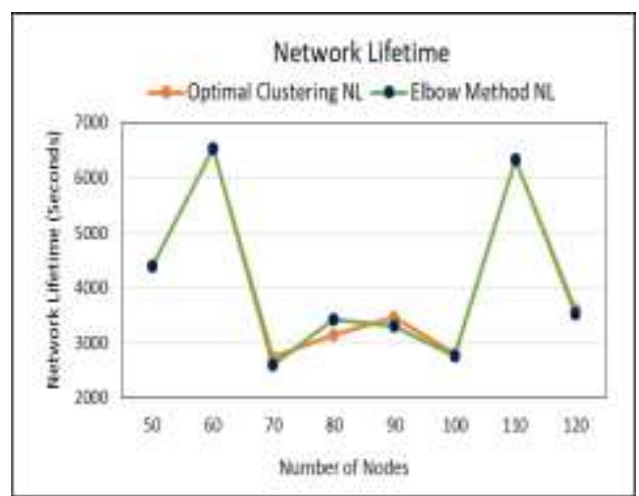

Figure 8. Comparison of Network Lifetime for Elbow and OC Method

Computational Intelligence and Communication Networks (CICN), IEEE(2010) (275-278)

2. Bhawna, Tanu Pathak and Virender Ranga, A Comprehensive Survey of Clustering Approaches in Wireless Sensor Networks, Elsevier Publications (2014) (685-691)

3. W.B. Heinzelman, A.P. Chandrakasan and H. Balakrishnan, An Application-Specific Protocol Architecture for Wireless Microsensor Networks, IEEE Trans. Wireless Communications, (1) (4) (2002)(660-670)

4. O. Younis and S. Fahmy, HEED: A Hybrid, Energy-Efficient Distributed Clustering Approach for Ad Hoc Sensor Networks, IEEE

Transactions on Mobile Computing, (3) (4)(258269)(2004)

5. P. Sasikumar and Sibaram Khara, K-Means Clustering In Wireless Sensor Networks, Fourth International Conference on Computational Intelligence and Communication Networks (140-144) (2012)

6. Purnima Bholowalia and Arvind Kumar, EBKMeans: A Clustering Technique based on Elbow Method and K-Means in WSN, International Journal of Computer Applications, (105) ( 9) (2014) DOI: 10.5120/18405-9674

7. Basma Fathi Solaiman and Alaa F. SHETA, Energy optimization in wireless sensor networks using a hybrid K-means P7SO clustering 
algorithm, International Journal of Computer Applications, (24) (4) (2013) (2679-2695)

8. Khan A., Tamim I., Ahmed, E., and Awal M. A., Multiple parameter based clustering (MPC): Prospective analysis for effective clustering in wireless sensor network (WSN) using K-means algorithm, Wireless Sensor Network, (4) (1) (2012) (18-24)

9. Adnan Alrabea, A. V. Senthilkumar, Hasan AlShalabi and Ahmad Bader, Enhancing K-Means Algorithm with Initial Cluster Centers Derived from Data Partitioning along the Data Axis with PCA, Journal of Advances in Computer Networks, (2) (2013)

10. Vinay Kumar, Sanjay B. Dhok, Rajeev Tripathi and Sudarshan Tiwari, A Review Study on Analytical Estimation of Optimal Number of Clusters in Wireless Sensor Networks, Transactions on Networks and Communications, (2) (5) (2014)(75-103)

11. H. Chen, C. K. Tse and J. Feng, Minimizing effective energy consumption in multi-cluster sensor networks for source extraction, IEEE Transactions on Wireless Communications, (8) (3) (2009) (1480- 1489)

12. Stan Salvador and Philip Chan, Determining the number of Clusters/Segments in Hierarchical Clustering/Segmentation Algorithms, 16thIEEE International Conference on Tools with AI (ICTAI),(2004) (576-584)

13. Chan T.J., Chen M. C., Huang Y.F., Lin J. Y. and Chen T.R., Optimal Cluster Number Selection in Ad-hoc Wireless Sensor Networks, WSEAS Transaction on Communication, (7) (8) (2008) (837-846)
14. Boris Mirkin, 'Choosing the number of clusters', Department of Data Analysis and Machine Intelligence, Wiley Interdisciplinary Reviews: Data Mining and Knowledge Discovery (1) (3)(2011) (252-260)

15. Chen H. and Megerian S., 'Cluster Sizing and Head Selection for Efficient Data Aggregation and Routing in Sensor Networks', Proceeding of IEEE WCNC, (4) (2006) (2318-2323)

16. L. C. Wang, C. W. Wang and C. M. Liu, Optimal number of Clusters in Dense Wireless Sensor Networks: A Cross-Layer Approach, IEEE Transactions on Vehicular Technology, (58) (2) (2009)(966-976)

17. Chunawale Abhishek and Sumedha Sirsikar, Minimization of average energy consumption to prolong lifetime of Wireless Sensor Network, In Wireless Computing and Networking, Global Conference on Wireless Computing and Networking (GCWCN), IEEE (2014) (244-248)

18. Shea, John M. and Joseph P. Macker, Automatic selection of number of clusters in networks using relative eigenvalue quality, Military Communications Conference, MILCOM 2013, IEEE (2013) (131-136)

19. Raghuvanshi, Ajay Singh, Sudarshan Tiwari, Rajeev Tripathi and Nand Kishor, Optimal number of clusters in wireless sensor networks: a FCM approach, International Journal of Sensor Networks, (12) (1) (2012) (16-24) 